\title{
Borst, Eva
}

\section{Gefährdungen des Denkens. Zum Verhältnis von Frauen und Macht}

Borst, Eva [Hrsg.]; Casale, Rita [Hrsg.]: Ökonomien der Geschlechter. Opladen ; Farmington Hills : Verlag Barbara Budrich 2007, S. 13-28. - (Jahrbuch Frauen- und Geschlechterforschung in der Erziehungswissenschaft; 3)

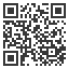

Quellenangabe/ Reference:

Borst, Eva: Gefährdungen des Denkens. Zum Verhältnis von Frauen und Macht - In: Borst, Eva [Hrsg.]; Casale, Rita [Hrsg.]: Őkonomien der Geschlechter. Opladen ; Farmington Hills : Verlag Barbara Budrich 2007, S. 13-28 - URN: urn:nbn:de:0111-opus-53587 - DOI: 10.25656/01:5358

https://nbn-resoiving.arg/um:nbn:de-0111-opus-53587

https://doi.org/10.25656/01:5358

in Kooperation mit / in cooperation with:

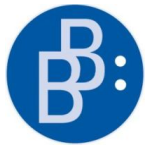

https://mww.budrich.de

\section{Nutzungsbedingungen}

Gewāhnt wird ein richt exklusives, nicht übertragbares, persōoliches und beschrărikes Recht auf Nulzung dieses Dokuments. Dieses Dokument ist ausschießlich für den persönlichen. nicht-kommerziellen Gebrauch bestimmt. Die Nutzung stelli keine Ubertragung des Eigentumsrechts an diesem Dokument dar und gill vorbehaltich der folgenden Einschränkungen: Aul sảmticthen Kopien deses Dokuments müssen ale Urheberrechtshirweise und sonstigen Hinweise aut gesetzlichen Schutz beibehallen werden. Sie dürlen dieses Dokument nicht in irgendeiner Weise abändern, noch dürlen Sie dieses Dokument für ölfentiche oder kommerzielle Zwecke verviellältigen, D̈flentlich ausstellen, auffuhren, vertreben oder anderweitig nutzen.

vertreben oder anderweitig nutzen.
Mil der Verwendung dieses Dokuments erkennen Sie die Nutzungsbedingungen an.

\section{Terms of use}

We grant a non-exclusive, non-transferable, individual and limited right to using this document.

This document is solely intended for your personal, non-commercial use. Use of this dooument does not include any transfer of property rights and it is conditional to the following limitations: All of the copies of this documents must retain all copyright information and other information regarding legal protection. You are not allowed to alter this document in any way, io copy it for public of commercial purposes, to exhibit the document in public, to perform, distribute of otherwise use the document in public.

By using this particular document, you accept the above-stated conditions of use.

\section{Kontakt / Contact:}

peDOCS

DIPF | Leibniz-Institut für Bildungsforschung und Bildungsinformation

Informationszentrum (IZ) Bildung

E-Mail: pedocs@dipf.de

Internet: www.pedocs.de

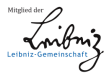


Eva Borst

Rita Casale (Hrsg.)

\section{$3 / 2007$}

Jahrbuch Frauen- und Geschlechterforschung in der Erziehungswissenschaft Ökonomien der Geschlechter 
Ökonomien der Geschlechter 
Jahrbuch der

Frauen- und Geschlechterforschung

in der Erziehungswissenschaft

herausgegeben von

Rita Casale

Barbara Rendtorff

Sabine Andresen

Vera Moser

Annedore Prengel

Beirat

Birgit Althans, Berlin

Eva Borst, Mainz

Eva Breitenbach, Osnabrück

Bettina Dausien, Bielefeld/München

Isabell Diehm, Bielefeld

Hannelore Faulstich-Wieland, Hamburg

Edgar Forster, Salzburg

Edith Glaser, Dortmund

Carola Iller, Heidelberg

Andrea Liesner, Hamburg

Susanne Maurer, Marburg

Inga Pinhard, Frankfurt

Folge 3/2007 
Eva Borst

Rita Casale (Hrsg.)

\section{Ökonomien der Geschlechter}

Verlag Barbara Budrich

Opladen \& Farmington Hills 2007 
Bibliografische Informationen der Deutschen Nationalbibliothek

Die Deutsche Nationalbibliothek verzeichnet diese Publikation in der Deutschen Nationalbibliografie; detaillierte bibliografische Daten sind im Internet über http://dnb.d-nb.de abrufbar.

Gedruckt auf säurefreiem und alterungsbeständigem Papier.

Alle Rechte vorbehalten.

(C) 2007 Verlag Barbara Budrich, Opladen \& Farmington Hills

www.budrich-verlag.de

ISBN 13: 978-3-86649-088-8 / eISBN: 978-3-86649-891-4

Das Werk einschließlich aller seiner Teile ist urheberrechtlich geschützt. Jede Verwertung außerhalb der engen Grenzen des Urheberrechtsgesetzes ist ohne Zustimmung des Verlages unzulässig und strafbar. Das gilt insbesondere für Vervielfältigungen, Übersetzungen, Mikroverfilmungen und die Einspeicherung und Verarbeitung in elektronischen Systemen.

Umschlaggestaltung: disegno visuelle kommunikation, Wuppertal - www.disenjo.de Satz: Beate Glaubitz Redaktion + Satz, Leverkusen

Druck: paper\&tinta, Warschau

Printed in Europe 


\section{Inhalt}

\section{Editorial}

\section{Essay}

Eva Borst

Gefährdungen des Denkens: Zum Verhältnis von Frauen und Macht

\section{Beiträge}

Rosemarie Ortner

Der Homo oeconomicus feministisch gebildet? Eine neoliberale

Herausforderung für das Subjektverständnis feministischer Bildungstheorie

Heike Kahlert

Emanzipatorisches Wissen im Schatten des Neoliberalismus:

Ökonomisierung der Kritik oder Kritik der Ökonomisierung?

Edgar Forster

Feminisierung und Geschlechterdifferenz.

\section{Work in Progress}

Stephanie Maxim

Geschlecht und Erfahrung: Zur Reifizierung von Geschlecht in der schulischen Geschlechterforschung

Angelika Paseka

Gender Mainstreaming und Transformationsprozesse im Bildungswesen:

Hoffnungen und Realitäten

Dorle Klika

Fächerwahl im Lehramtsstudium - Zementierung der

Geschlechtersegregation? 
Karin Priem

Almersia 1994-2003: Migration und biografisches Bilderwissen in

Fotografien von Rineke Dijkstra

Sylvia Bürkler

Die Genfer Frauenrechtlerinnen zu Beginn des 20. Jahrhunderts

\section{Rezensionen}

Christine Rabl

Rezension zu Mona Singer: Geteilte Wahrheit. Feministische Epistemologie,

Wissenssoziologie und Cultural Studies, Wien: Löcker 2005

Bettina Hünersdorf

Rezension zu Anja Tervooren: Im Spielraum von Geschlecht und Begehren.

Ethnographie der ausgehenden Kindheit, Weinheim: Juventa 2006

Susanne Maurer

Rezension zu Barbara Rendtorff: Erziehung und Geschlecht. Eine

Einführung, Stuttgart: Kohlhammer 2006

Karin Manz

Rezension zu Anne Schlüter (Hg.): Bildungs- und Karrierewege von Frauen. Wissen-Erfahrung-biographisches Lernen, Opladen: Verlag Barbara Budrich 2006

Marcus Rieger-Ladich

Rezension zu Sabine Hark: Dissidente Partizipation. Eine

Diskursgeschichte des Feminismus, Frankfurt am Main: Suhrkamp 2005

Sabina Larcher

Rezension zu Marina d'Amelia: La Mamma, Bologna: Società editrice il Mulino 2005

Rebekka Horlacher

Rezension zu Yvonne Leimgruber: In pädagogischer Mission. Die Pädagogin Rosette Niederer-Kasthofer (1779-1857) und ihr Wirken für ein „frauengerechtes“ Leben in Familie und Gesellschaft, Bad Heilbrunn: Klinkhardt 2006

\section{Ankündigung der nächsten Bände}

Jahrbuch Band 4 


\section{Gefährdungen des Denkens Zum Verhältnis von Frauen und Macht}

\section{Eva Borst}

Geschichte - Im Unterschied zu heutigen wissenschaftlichen Verlautbarungen aus männlicher Feder hatte zur Zeit des Neuhumanismus' Geschlecht einen systematischen Ort im philosophischen Denken, wenn auch von heute aus gesehen einen zumeist wenig schmeichelhaften. Als Beispiel mag Wilhelm von Humboldt gelten, der ausdrückl ich die Gesc hlechterdifferenz als Muster für di e Mannigfaltigkeit der menschlichen Kräfte heranzieht und an i hr die Wechselwirkung gegeneinander strebender Kräfte demonstriert. Es wi rd wohl kaum überraschen, dass Humboldt dabei dem zu seiner Zeit dominanten Geschlechterdiskurs folgt und dem Männlichen mehr zeugende, selbsttätige, aufklärende Wirkung zuschreibt, indes er dem Weiblichen mehr empfangende, verharrende, empfindende Kräfte attestiert und diese mit Verhaltensdispositionen in Verbindung bringt. Frauen haben demnach weni ger Interesse an abstrakten Spekulationen, wie sie überhaupt eher zur Einbildungskraft neigen und dem Unmittelbaren, dem Subjektiven mehr verhaftet sind. Ihre intellektuellen Fähigkeiten erschöpfen sich Humboldt zufolge in der B etrachtung der Natur, dem Symbolischen und Mittelbaren gegenüber verspüren sie gar eine Abneigung. Im Grunde handelt es sich um ei nen altbekannten Sachverhalt, der der Erwähnun g kaum bedürfte, würde hier nicht etwas in Szene gesetzt, das bis heute nachhaltigen Einfluss auf di e Wahrnehmung der wei blichen Wissensfähigkeit und Wissenschaftsfähigkeit hat und diese steuert. Auch wenn heutzutage mehr Professorinnen als jemals zuvor Lehrstühle besetzen, und damit der Anspruch der Frauen auf Wissen und Erkennt nis deutlich sichtbar an Konturen gewonnen hat, lässt sich doch nicht leugnen, dass man sie auf diesen machtvollen Positionen keineswegs willkommen heißt. Wie anders wäre es sonst zu erklären, dass es trotz verfassungsmäßig garantierter Gleichheit, trotz Gleichstellungsbeauftragter und $t$ rotz finanzieller Anreizsysteme nicht gelingen will, ihnen den gleichberechtigten Einzug in die Universitäten zu gewähren. An habilitierten Frauen jedenfalls fehlt es nicht.

Die Ursache für dieses prekäre Verhältnis liegt also tiefer, als es auf den ersten Blick erscheinen mag und resultiert aus einer historischen Hypothek, 
die insbesondere in Humboldts Urteil über die Wissensfähigkeit der Frauen zum Ausdruck kommt. Das allen Frauen innewohnende Muttergefühl und die damit verbundene unhintergehbare Fähigkeit zur Aufopferung verlangt, so Humboldt, eine andere Form der Aneignung, eine andere Form, sich mit der Welt vertraut $\mathrm{zu}$ machen, verlangt eine Bildung, die niemals den engen $\mathrm{Zu}-$ sammenhang zwischen Körperlichkeit und Intellektualität vergessen machen darf. Theoretische Entwürfe, abstrakte Modelle oder etwa transzendentale Konzepte werden von ihnen nicht erwartet.

Weibliche Intellektualität - Das repräsentative Sprechen eines intellektuellen Mannes über Frauen im imaginären Bewusstsein, die Wahrheit zu sagen, konvergiert mit der Gewissheit, dass die Wahrnehmung der Welt bei den Geschlechtern auf unterschiedliche Weise von statten geht. So wird die Verteilung des Wissens aus der Position der Macht einer geschlechtsspezifischen Codierung unterworfen, die zugleich konstitutiv ist für die weibliche Identitätsbildung und Auswirkungen bis in unsere heutigen Tage zeitigt. Selbst wenn Frauen heute für sich sprechen und ihnen im Zeichen einer demokratisch verfassten Grundordnung formal der Anspruch auf Selbstbestimmung zusteht, so b leibt die gesellschaftliche Machtverteilung noch immer an die Kategorie Geschlecht gebunden. Die Zähigkeit, mit der sich dieses Muster hält, hat ihre Ursache zum einen im kommunikativen Gedächtnis der bürgerlichen Familie, in der die historischen Diskurse über den Geschlechtsunterschied lebendig geblieben sind und sich unreflektiert fortsetzen. Zum anderen ist sie Resultat der symbolischen Ordnung, für die das repräsentative Sprechen geradezu konstitutiv ist, denn der Vater ist derjenige, der über das Wissen und die Macht verfügt, die Ordnung aufrecht zu erhalten. Wie Freud und Lacan gezeigt haben, „bringt sich im Rahmen der patriarchalen Kultur der Vater als symbolische Machtinstanz hervor, indem durch ihn das imaginäre Ganzheits- und Einheitsphantasma der Mutter-Kind-Dyade unterbrochen" und ein grundlegender Mangel erzeugt wird, der sich in der Trennung vom mütterlichen Körper offenbart und durch den ,phallisch fetischisierten Körper des Vaters ersetzt wird“ (Schuller 1990, S. 190). Mit d em Eintritt in die symbolische Ordnung ist aber nicht nur die Trennung vom mütterlichen Körper und zugleich die Anerkennung des „väterlichen Wissens“ in Gestalt der sozialen Gesetze, der Sprache und der Werte vollzogen. Vielmehr wird dieser Mangel im Moment seiner Entstehung schon verleugnet und zum Zeichen der weiblichen Geschlechtsidentität. Ermöglicht die Identifikation mit dem symbolischen Vater als Repräsentant der Macht die männliche Geschlechtsidentität, so konstituiert sich die weibliche durch eine unhintergehbare Differenz 
zum symbolischen Vater, die sie aufgrund des bestehenden Wertekanons als inferior ausweist und sie von der Macht ausschließt. Dieser Prozess kann keinesfalls als ein voluntaristischer Akt vorgestellt werden, weil wir schon im Augenblick der Geburt von der symbolischen Ordnung eingefasst sind und nicht entrinnen können, es sei denn auf Kosten der Sprach- und Kulturfähigkeit und damit auf Kosten der weiblichen Intellektualität selbst.

Im Hinblick auf die geschlechtsspezifische Codierung des Wissens bzw. der Wissensfähigkeit und hinsichtlich des damit einhergehenden Machtverlustes auf Seiten der Frauen ergibt sich aus dem genannten Unterschied zum männlichen Geschlecht ein interessanter Aspekt, den Marianne Schuller in ihrer Studie zur Vergabe des Wissens luzide und scharfsinnig herausarbeitet. Der nun schon über 20 Jahre alte Text eröffnet Einsichten, die nach wie vor aktuell sind, weil sich an den gesellschaftlichen Tiefenstrukturen kaum etwas verändert hat, die politischen Interventionen der Frau en aber in der Zwischenzeit nahezu vollständig zum Stillstand gekommen sind. Einerseits weil die Frauenbewegung in gewisser Hinsicht erfolgreich bei ihrem Kampf um die rechtliche Emanzipation war. Andererseits aber hat gerade dieser Erfolg $\mathrm{zu}$ ihrer Bürokratisierung geführt und sie im Wesen tlichen stumpf werden lassen gegen immer noch bestehende offensichtliche und subtile Benachteiligungen und Diskriminierungen. Schlimmer noch: Ihre originären Themen, wie etwa der Kampf gegen sexualisierte Gewalt gegen Mädchen und Frauen ist in der Zwischenzeit zum Quotenhit der Fernsehanstalten verkommen, wie unlängst der Fall Kampusch in aller Deutlichkeit gezeigt hat, ohne dass etwa über Gewalt als solche öffentlich diskutiert und sie gesellschaftlich geächtet würde. $\mathrm{Ob}$ das bürokratische Ungeheuer Gendermainstreaming an diesen Strukturen zu rühren vermag, ist zu bezweifeln.

Schuller plädiert nun in ihrer Studie für eine radikale Wissens- und Machtkritik, die sich gerade an der Differenz zum symbolischen Vater entzündet. Auch wenn sich die Weiblichkeit an der mütterlichen Position bilde und sich über den Ausschluss von der Macht hervorbringe, so sei sie doch unhintergehbar der Reproduktionsfundus, dem sich die Macht verdanke. In dieser Funktion sei sie nicht nur Opfer der symbolischen Ordnung, sondern zugleich deren stumme und verschwiegene Stütze. Allein schon die Rekonstruktion dieses Sachverhalts sei ein Angriff auf die Macht, die in dem Maße wirke als sie im Mythos des „Naturwüchsigen“ verschwinde (vgl. Schuller 1990, S. 191). Diese Abhängigkeit überhaupt ins Bewusstsein zu rufen, erzeuge eine Instabilität, die die Voraussetzung dafür sei, intellektuell fundierte Widerstandsformen zu entwickeln, die an den Wurzeln der Machtkonstitution selbst rühren. Mag auch für intellektuelle Frauen die Bedingung der Mög- 
lichkeit geschaffen sein, das Begehren nach Macht und Wissen zu artikulieren, so bleibt doch ihre Position stets eine prekäre.

Die performative Erfüllung der ,väterlichen Ordnung“ ist Voraussetzung für ihr Funktionieren, daher wird das „väterliche Wissen“ in der männlichen Genealogie weitergegeben und in wechselseitigen homoerotischen Anerkennungsprozeduren gefestigt. Werden Frauen hingegen als Intellektuelle wahrgenommen, so geht dieser Anerkennungsprozess in doppelt verkennender Weise und meist auf Kosten der eigenen weiblichen Ich-Identität von statten: Die „Frau wird zu dem Mann, der sie nicht ist“ (Schuller 1990, S. 189). Da Frauen aus der Position der symbolischen Ordnung Intellektualität nicht zusteht, ist mit ihrer Anerkennung durch die Macht zugleich auch die Anerkennung der Macht verbunden. Mit anderen Worten: Weibliche Intellektualität ist stets der Gefahr ausgesetzt, zur Stabilisierung der symbolischen Ordnung beizutragen und damit als verschwiegene Stütze zu fungieren. Gleichzeitig wohnt ihr ein k ritisches Potenzial inne, ein Rest, der nicht von der Macht vereinnahmt werden kann: die weibliche Geschlechtsidentität. Indem sie nämlich ,als verschwiegene Stütze die väterliche Macht mitkonstituiert, kann sie von dieser nicht aufgenommen werden" (Schuller 1990, S. 193).

Die Spiele der Macht aber sind vielfältig, denn der Kampf um Anerkennung führt zu unterschiedlichen Verhaltensweisen. So variieren die Szenerien um die Gunst der Macht zwischen Koketterie und sexueller Attraktivität, zwischen Verleugnung der eigenen Potenzialität und einem zutiefst konkurrenten Verhalten. Allen diesen Spielarten ist eines gemeinsam: Sie stellen die Ordnung als solche nicht in Frage. Im Gegenteil bestätigen sie erst das, was sie eigentlich zu unterlaufen trachten. Angesichts dieser Situation bleibt als Gegenbewegung die Möglichkeit, im Intellektualitätsprozess selbst Wi derstandsformen zu entwickeln, die den Ort der Entstehung der weiblichen Geschlechtsidentität und damit den Konstituierungsprozess der väterlichen Macht zum Ort der Unruhe machen und die subtilen Strategien, weibliche Intellektualität im Sinn e der symbolischen Ordnung zu vereinnahmen, aufdecken. Den Konstitutionsprozess selbst zu stören kann allerdings nicht diesseits oder jenseits des ,väterlichen Wissens“ geschehen, sondern in der Kritik dieses Wissens. Das schließt notwendig eine Institutionenkritik mit ein.

Schullers Notizen zum Verhältnis von „weiblicher Intellektualität" und Macht sind durchaus nicht als Gedankenexperiment zu verstehen, sondern als theoretisch-politische Forderung, die nach praktischen Konsequenzen verlangt. Das Projekt scheint allerdings vorläufig gescheitert. Denn wenn PolitikerInnen wieder ganz ungeniert von der bürgerlichen Familie vergangener Jahrhunderte schwärmen, PublizistInnen das Streben der Frauen nach Er- 
werbsarbeit als biologische Verirrung abtun und WissenschaftlerInnen den Geschlechtsunterschied als naturgegeben vorstellen, dann steht dahinter das bislang noch weitgehend unausgesprochene Eingeständnis, dass 30 Jahre avancierter Frauen- und Geschlechterforschung nicht vermochten, an den Fundamenten gesellschaftlicher Geschlechterungleichheit zu rütteln. Mehr noch: ihre Ergebnisse werden ignoriert. In der Erziehungswissenschaft zeigt sich dies etwa darin, dass die Autoren des 12. Kinder- und Jugendberichts 2005 auf hirnphysiologisch motivierte Studien zurückgreifen, die davon ausgehen, dass das weiblich Gehirn empathisch, das männliche hingegen systematisch sei und damit schon überwunden geglaubte Vorurteile perpetuieren. Das nötigt zu der Einsicht, dass hier offenbar ein Deutungsmuster in Gang gesetzt ist, das sich in machtvollen, Wissen produzierenden und formierenden Diskursen niederschlägt und in einer Geste der Verwerfung die ReNaturalisierung des Geschlechtsunterschieds betreibt, nicht zuletzt im Interesse einer zynischen Biopolitik, die Frauen für den demographischen Wandel verantwortlich macht und ihnen die Last der Reproduktionsarbeit einmal mehr aufbürdet. Die darin zum Ausdruck kommende Verschränkung von populären Meinungen, wissenschaftlichem Expertentum und politischen Wunschvorstellungen hat Pierre Bourdieu kurz vor seinem Tod scharf angegriffen. In seinem Vortrag „Der Beruf der Wissenschaft und die soziale Bewegung“ erinnert er nachdrücklich daran, dass einer der theoretischen und praktischen Irrtümer vieler Theorien derjenige gewesen sei, die Wirksamkeit von Theorie außer Acht gelassen zu haben. Diese Wirksamkeit gründet seiner Auffassung nach in der von jeder Theorie ausgehenden Autorität, deren Träger sich allerdings in vermeintliche oder tatsächliche Experten ausdifferenzierten. Die vermeintlichen Experten geben sich Bourdieu zufolge lediglich einen „wissenschaftlichen Anstrich" (Bourdieu 1998, S. 61), um auf diese Weise in medial aufbereiteten Botschaften politische Autoritätseffekte zu erzielen, die ihnen diskursive und nicht-diskursive Machtvorteile verschafften. Mit dieser Analyse zieht er jedoch keinesfalls den Wert oder die Aussagekraft von Theorien in Zweifel. Im Gegenteil gilt sein Plädoyer einer verschärften theoretischen Auseinandersetzung und der Frage, ob das, was in offiziellen politischen Stellungnahmen als vernünftig gilt, tatsächlich vernünftig im Sinne sozialer Gerechtigkeit ist. Er reiht sich damit in die Reihe derjenigen Intellektuellen und tatsächlichen Experten ein, die aus der Perspektive wissenschaftlicher Kritik gesellschaftliche Umbrüche kommentieren.

Aber auch wenn Intellektuelle aus der Position der Kritik heraus argumentieren, so bleiben sie doch Teil des Kritisierten, sie bleiben Teil des Machtsystems, dem sie sich zugleich verdanken. Marianne Schuller hat gera- 
de diesen Umstand im Auge, wenn sie von der weiblichen Intellektualität als einer radikal kritischen Instanz spricht, die die Kritik der Kritik nicht ausschließt. Danach ist die Wissenschaft gehalten, sich stets aufs Neue in Frage zu stellen, auch die Geschlechterforschung selbst.

Da das handelnde, dabei intelligible Subjekt Effekt und Umschlagplatz von Macht ist, erhält ihre Forderung nach einer theoretisch-politischen Wissenskritik eine praktische Komponente, insofern Theorie selbst Praxis ist. In den Worten Foucaults: „Heute kommt es dem Intellektuellen aber nicht mehr zu, sich an die Spitze oder an die Seite aller zu stellen, um deren stumme Wahrheit auszusprechen. Vielmehr hat er dort gegen die Macht zu kämpfen, wo er gleichzeitig deren Objekt und deren Instrument ist: in der Ordnung des ,Wissens', der ,Wahrheit', des ,Bewußtseins', des Diskurses. Darum ist die Theorie nicht der Ausdruck, die Übersetzung, die Anwendung einer Praxis; sie ist selbst eine Praxis. Aber eine lokale und regionale Praxis, die [...] nicht totalisiert. Sie ist Kampf gegen die Macht, Kampf um ihre Sichtbarmachung und Schwächung dort, wo sie am unsichtbarsten und hinterhältigsten ist" (Foucault 1987, S. 108). Theorie dient demnach keinem Selbstzweck und schon gar nicht einer irgendwie gearteten Wahrheit. Sie ist das Instrumentarium, Macht sichtbar zu machen. Demzufolge ist ein theoretisches Denken in dem Moment ein politisches Denken, wenn es sich in ein er unaufhörlichen Bewegung auf seine eigenen Konstitutionsbedingungen zurückzuwenden vermag und in dieser Rückwendung zugleich kulturell vorgeformte Erkenntnisraster aufdeckt und verworfenem, ausgeschlossenem oder an den Rand gedrängtem Wissen Bedeutung verleiht. Insofern bringt sich weibliche Intellektualität stets an den Grenzen der Formierungszwängen einer heterosexuellen Gesellschaft hervor und durchkreuzt „die identitätslogische Monumentalität des ,väterlichen Wissens““ (Schuller 1990, S. 194).

Die Universität - Intellektuelle Gesten in Gestalt von Vorträgen und Publikationen sind nicht an eine Institution wie die Universität gebunden, gleichwohl aber ist sie es, die den materiell abgesicherten Ort für ein kreatives Denken zur Verfügung stellen sollte. Dies erstens, weil nur in einer von wirtschaftlichen und politischen Interessen freien Situation Denken überhaupt möglich ist. Denken nämlich verzehrt Zeit, es unterbricht das Tun und ist nicht immer auf praktische Zwecke hin ausgerichtet, da Abirrungen, Täuschungen und die Wahrnehmung verwandter oder abweichender Ansichten geradezu konstitutiv für den Denkprozess selbst sind. Unter diesen Voraussetzungen beansprucht Denken eine Ökonomie, die seinen zuweilen unvorhersehbaren Strukturen ausreichend Raum und Zeit zur Entfaltung lässt. 
Denken, so schreibt Hannah Arendt, bedeutet den Rückzug aus dem Alltagsgeschäft, und daran hat sich bis heute nichts geändert. Daraus ergibt sich zweitens, dass die Universität trotz ihrer Öffnung in die Gesellschaft hinein ein Ort bleiben muss, an dem es möglich ist, ohne ein beständiges Schielen auf die Verwertbarkeit der eigenen Forschungsleistung geistig produktiv zu bleiben und kritische Kommentare gegen den Zeitgeist öffentlich zu verlautbaren. Obwohl dieses außergewöhnliche und unerlässliche Privileg der Universität heute politisch nicht mehro pportun und einer naiven, bisweilen geistlosen Sorge um den Wirtschaftsstandort Deutschland gewichen ist, erscheint es gleichwohl angebracht, gerade diese Institution, die offiziell zu betreten Frauen in Deutschland erst seit rund 100 Jahren gestattet und in der $\mathrm{zu}$ forschen und zu lehren ihnen sogar erst seit rund 87 Jahren erlaubt ist, gegen den Geist einer wirtschaftsliberalen Elite zu v erteidigen. Auch wenn die Universität in der Zw ischenzeit ihren Anspruch auf Deutungshoheit eingebüßt hat, so kommt ihr doch eine Funktion zu, ohne die eine entwickelte Demokratie nicht existieren kann: Der Widerspruch, das Streitgespräch, die Kritik, der Zweifel oder wie Derrida schreibt, das Recht, ,alles zu sagen, sei es auch im Zeich en der Fiktion, und der Erprobung des Wissens" und das Recht „es öffentlich zu sagen“ (Derrida 2001, S. 14). Es sind diejenigen Koordinaten, die der Aufklärung verpflichtet bleiben und zu einem zivilgesellschaftlichen Konsensus unerlässlich sind.

Der politisch forcierte Eingriff der Wirtschaft in die akademische Kultur - gesteuert von supranationalen Organisationen wie der OEC D, gefördert durch Unternehmensberatungen wie McKinsey und unterstützt durch das private, Bertelsmann eigene Centrum für Hochschulentwicklung (CHE) - hat fatale Folgen auch für den akademischen Feminismus und die Geschlechtertheorie, weil sie als w enig produktiv für die neuen Aufgaben der Universität wahrgenommen werden. In Zeiten, in denen sich Geistes-, Sozial- und Gesellschaftswissenschaften auf ihre Effizienz im Hinblick auf den Arbeitsmarkt befragen lassen müssen, wird eine Forschung, die grundsätzliche Probleme im Modus der Kritik formuliert, als Ballast empfunden. Nicht zuletzt deshalb, weil sie, ihrem wissenschaftlichen Verständnis nach, dort wirksam wird, wo die Macht sich mit dem Schleier der Unsichtbarkeit umgibt und unbemerkt sich zu totalisieren versucht.

Gerade die Akademisierung einer vormals sozialen Bewegung hat nicht nur dazu beigetragen, Geschlecht als Analysekategorie in das akademische Bewusstsein zu heben, nein, ihr ist es a uch nach der gewaltvollen Zäsur durch die Nationalsozialisten gelungen, die Geschichte unter geschlechtsspezifischer Perspektive neu auszuleuchten, zu dokumentieren und dereinst gül- 
tige Wahrheitsansprüche einer grundlegenden Revision zu unterziehen. Eines der wegweisendsten Ergebnisse war die Denaturalisierung des Geschlechtsunterschieds. Im Rahmen von äußerst ambitionierten, dabei kontroversen Auseinandersetzungen gelang es schließlich intellektuellen Frauen, das ,väterliche Wissen“ zu dekonstruieren, beispielsweise die Zwangsheterosexualität und die damit verbundenen Normalisierungsstrategien. Diese fruchtbaren Debatten über Begriffe, Modelle und Theorien in den unterschiedlichen Disziplinen sind Teil einer akademischen Kultur, die, um noch einmal mit Derrida zu sprechen, im ganz wörtlichen Sinne die Humanities auszeichnen. Aufzufassen wären demnach Fragen nach Recht und Gerechtigkeit als die „Problematisierung [...] jener wirkungsmächtigen juridischen Performative, die die moderne Geschichte dieser Menschlichkeit des Menschen skandieren“ (Derrida 2001, S. 67). Und dazu gehören eben auch, wie Derrida ausdrücklich betont, die Declaration des droits de l'homme - et de la femme, es gehört dazu die Aufklärung von Verbrechen gegen die Menschlichkeit im internationalen Maßstab. Das heißt, die Problematisierung dieser uralten Frage nach dem, was Menschlichkeit bedeuten kann, sollte heute wieder mehr denn je diskutiert werden, und zwar unter Bedingungen, die ein Denken in einer unbedingten Unabhängigkeit ermöglichen. Dieser der Aufklärung geschuldete Imperativ folgt dabei dem Prinzip, die Geschichte, die Methodologien und die Theorien der eigenen Disziplin, kurz das Denken selbst einer Befragung zu unterziehen. Ja der klassische Ort des Denkens selber, die Universität, darf davon nicht unberührt bleiben: „Dieses Prinzip unbedingten Widerstands ist ein Recht, das die Universität selbst zugleich reflektieren, erfinden und setzen müsste" (Derrida 2001, S. 13) Dass dieser Satz mit einem Konjunktiv endet, kann kaum überraschen, denn die Zukunft der Universität wird zunehmend von gesellschaftlichen Kräften bestimmt, die die Humanwissenschaften, so auch die Erziehungs- und Bildungswissenschaft, dem eigenen Verwertungsinteresse unterwerfen und dem Glauben anhängen, vermittels wirtschaftlicher Controllingverfahren die Wissensproduktion beschleunigen und steu ern zu können.

Ökonomisierung und Beschleunigung, Kontrolle und Steuerung des Wissens im Namen des Neoliberalismus verschärft die Situation für den akademischen Feminismus auf eine doppelt problematische Weise: Erstens ist die zunehmende Ökonomisierung der Universität und das anvisierte beschleunigte Durchlaufen des Bildungssystems ja nicht nur ein von außen gesteuerter Oktroy. Im Gegenteil beteiligen sich die Universitäten rege an der neoliberalen Umstrukturierung und liefern die dazu notwendigen theoretischen Inputs. Die in Mode gekommene Evolutionstheorie, die sich erkenntnistheoretisch 
im radikalen Konstruktivismus niederschlägt, verkommt unter neoliberalem Einfluss zur Legitimationsgrundlage eines marktkapitalistischen Wirtschaftssystems, dessen weltweite Durchsetzungskraft als ein Indiz für die Unhintergehbarkeit des Marktes als Regulierungsmechanismus ökonomischer und sozialer Beziehungen angesehen wird. Der Mythos der Naturwüchsigkeit allerdings rekurriert dabei auf eine essentialistisch-biologistische Anthropologie, von der sich insbesondere der Geschlechtsunterschied in seiner scheinbaren natürlichen Verfasstheit ableiten lässt. Die Resignifizierung der Biologie im Gewande der Evolutionstheorie sowie der Hirnphysiologie in Allianz mit der abgeforderten Anpassung an ökonomische Bedingungen schließt ein Denken im Sinne Derridas aus. Denn die substanzlogische Annahme einer ursprünglichen Natur, die sich uns gleichsam rein und ungebrochen präsentiert, insofern ihr vorkultureller Status alternativlos und vermeintlich zweifelsfrei festgestellt wird, beruht auf der reichlich naiven Vorstellung, es gebe so etwas wie ein unhintergehbares Faktum, auf das sich jede wissenschaftliche Analyse vorurteilsfrei beziehen lasse. Damit wird nicht nur die Geschichtlichkeit allen Wissens bestritten, sondern das Denken selbst unterwirft sich diesen Prämissen, es bleibt stets an eine Natu r gebunden, die es letztlich aber nicht ist. Diese äußerst effiziente Strategie, sich einer vorgeblich überzeitlichen Natur zu vergewissern, die die Notwendigkeit einer argumentativen Begründung überflüssig macht, relativiert nicht nur die gesellschaftlich-historische Verantwortung für ein bestimmtes Verhalten jedes Einzelnen, sondern sie eskamotiert darüber hinaus die Macht, die jedem hegemonialen Diskurs zugrunde liegt. Die Wahrnehmung dessen, was Frauen und Männer als Subjekte konstituiert, wird dadurch nachhaltig beeinflusst, ohne aber noch als machtvoll in Erscheinung zu treten.

Zweitens scheint Denken als Erkenntnisinstrument heute obsolet zu werden. So versteigt sich mitunter auch manch deutscher Professor zu der Behauptung, wer denke, forsche nicht, um geschwind wieder zu seinen empirischen Studien zu enteilen. Gerade aber die Universität hat Frauen die Möglichkeit geboten, theoretische Anordnungen wissenschafts- und machtkritisch zu hinterfragen und die eigenen Voraussetzungen einer Analyse zu unterziehen. Heute sind sie aber gehalten, ihre Lehre und Forschung, ihre Theorien und Methodologien zu legitimieren. Das wäre weiter nicht schlimm schließlich muss sich jegliches Denken Rechenschaft vor sich selbst ablegen -, würde diese Aufforderung nicht schon im Rahmen neoliberaler Vorgaben erfolgen und damit die Freiheit zur unbedingten Unabhängigkeit einschränken. Der Anpassungsdruck auf Wissenschaftlerinnen verschärft sich auf diese Weise. Denn nicht nur, dass sie die Kraft aufbringen müssen, in Abgrenzung 
zu jenem symbolischen Ort, der ihnen Macht und Wissen nur unter bestimmten Bedingungen zuteilt, ihre weibliche Intellektualität hervorzubringen, sind sie zusätzlich gehalten, ihr Lehr- und Forschungsangebot an die inhaltliche Neuausrichtung der Universität anzugleichen. Diese identitätslogische Nötigung zur Assimilation an den Arbeitsmarkt gewinnt ihre höchst problematische Seite insb esondere im g leichsam existentiellen Zwang zur Konkurrenz, der durch vielfältige Kontrollmechanismen erzeugt und aufrecht erhalten wird. Einer der entscheidendsten Faktoren in diesem Zusammenhang ist nicht die zunehmende Abhängigkeit von Drittmitteln, sondern die damit verbundene Forderung, immer schneller verwertbare Ergebnisse liefern zu müssen.

Diese Gemengelage produziert eine höchst instabile Situation. Zumal eine evolutionstheoretisch begründete Wirtschaftsordnung, in die Gesellschaft und Universität eingezogen sind und der sie unterliegen, unter den Voraussetzungen von Beschleunigung, Kontrolle und Steuerung zutiefst einem Denken widerspricht, das sich einem ethisch-moralischen Moment verdankt, wie es sich in den Humanities unter den Bedingungen der Menschenrechte entfaltet. Denken erschöpft sich nicht im Wissen und Verstehen, es ist die Tätigkeit, die das Wissen und Verstehen in Frage stellt und sich mit der „lebendigen Erfahrungen" (Arendt) verbindet.

Das Denken - Von Hannah Arendt erschien 1968 eine Reihe von Essays in einem Sammelband, den sie mit dem Untertitel „Übungen im politischen Denken“ versah. Ihr einziges Ziel sei es, so schreibt sie, „Erfahrung darin zu erwerben, wie man denkt" (Arendt 1994, S. 18). Es geht ihr darum, an der eigenen, individuellen Denkerfahrung den Prozess des Denkens selbst zu ergründen und die Tätigkeit des Denkens mit der lebendigen Erfahrung zu verknüpfen. Nur im Wechselverhältnis von Denken und Erfahrung wird demnach Denken zu einer Instanz, die uns in die Lage versetzt, uns in der Welt zurecht zu finden. Entsprechend notiert sie: ,[...] meine Annahme ist, daß das Denken aus Geschehnissen der lebendigen Erfahrung erwächst und an sie als die einzigen Wegweiser, mit deren Hilfe man sich orientiert, gebunden bleiben muß" (Arendt 1994, S. 18).

Ohne Erfahrung hätten wir keine Vorstellungen vom Leben, wir hätten keine Vorstellung von unserer Vergangenheit und schon gar nicht von einer zwar kontrafaktischen, noch zu erwartenden Zukunft. Erfahrung gelangt aber erst dann in unseren reflexiven Wahrnehmungshorizont, wenn sie als ein bedeutungsvolles Geschehnis rekonstruierbar wird. Nicht etwa der Inhalt der früheren Erfahrung, sondern ihr besonderer Bedeutungsgehalt für gegenwär- 
tige Problemstellungen ist das, was ihr Geltung verleiht. Gewinnt sich die Erfahrung also erst in der Rückbezüglichkeit, so erhält sie ihren Sinn in dem Moment, in dem sie zum Wegweiser unserer Handlungen wird. Da uns nur das Denken selbst in den Stand versetzt, ein Urteilsvermögen zu entwickeln, sofern es uns die Möglichkeit eröffnet, Unterscheidungen zu treffen, ist es zugleich die Voraussetzung für konkrete Entscheidungen. Dabei ist die Erfahrung diejenige Größe, auf die erinnernd reflektiert wird.

Von besonderem Interesse nun ist für Arendt die Lücke in der Zeit oder, wie sie an anderen Stellen schreibt: die Lücke zwischen Vergangenheit und Zukunft, die auszufüllen jeder Generation und jedem menschlichen Wesen von neuem und in geschichtlicher Verantwortung aufgegeben ist. Ausgangspunkt ihrer Überlegungen ist, dass das Denken das natürliche Bedürfnis eines jeden Menschen sei und nicht nur ein wissenschaftliches Unternehmen. Sie unterscheidet daher ein Denken, das zweckgebunden und im Hinblick auf eine Erkenntnis hin ausgerichtet ist, von einem Denken, das dem Leben Sinn verleiht und dazu befähigt, sich vor sich selbst Rechenschaft abzulegen. Denken und Erkennen sind demnach nicht dasselbe, oder anders ausgedrückt: Denken setzt zunächst keinen Zweck voraus, mehr noch: seine Ergebnisse bleiben ungewiss, nicht verifizierbar. Gleichwohl aber ist Arendt zufolge das Denken diejenige Tätigkeit, die uns erst befähigt, zwischen Recht und Unrecht zu unterscheiden und sich zu politischen Ereignissen in einer bestimmten Weise zu verhalten.

Das Denken fi ndet Arendt zufolge nur i n der Zwiesprache, in einem „stummen Dialog“ statt, in dem der Mensch sich seines Anderen versichert und in sein Eins-Sein einen Unterschied einfügt. Erst in dieser Alterität kann er sich zu sich selbst in Beziehung setzen, erst diese Alt erität ermöglicht überhaupt Denken: „Was das Denken in seinem Prozeß ak tualisiert, ist der Unterschied, der im Bewusstsein gegeben ist“ (Arendt 1994, S. 151). Mit Arendt kann man nun davon ausgehen, dass diese Alterität eine Denkerfahrung ist, in der wir uns als Individuum Rechenschaft darüber ab legen, ob wir richtig oder fal sch gehandelt haben, ob wir besser etwas unterlassen hätten oder ob wir etwas anderes hätten tun sollen, und zwar jenseits einer gesellschaftlichen Moral, die sich im Einzelnen betrachtet durchaus als unmoralisch erweisen kann. Dass sich das Denken erst in einem Prozess der Alterität konstituiert, ungewiss und nicht verifizierbar ist, bedeutet also nicht, dass es dem rei nen Selbstzweck dient, sondern Denken erzeugt als Nebenprodukt eine Moral, die sich der Frage nach dem richtigen Handeln verdankt (vgl. Arendt 1994, S. 94). Denken und moralisches Urteilen sind auf di ese Weise unhintergehbar miteinander verbunden. Aus dieser Konstellation könnte man schließen, dass die Moral als Produkt des 
Denkens dem Menschen eigentümlich ist. Die Feststellung aber, dass die Moral eine conditio sine qua non des $\mathrm{M}$ enschen darstellt, wirft die Frage auf, woher diese Moral kommt und welcher Maßstab der nämliche ist, um morali sch zu urteilen. Arendt hat darauf keine überzeugende Antwort gegeben. Denn wenn sie davon ausgeht, dass der Mensch ein moralisches Wesen ist, das sich in einem inneren Dialog Rechenschaft abzulegen vermag, dann stellt sich die Frage nach ihrer Herkunft nicht mehr. Sagen wi $r$ indes im Anschluss an Foucault, dass Moral diskursiv vermittelt ist, dann eröffnet sich zumindest die Möglichkeit, Moral als eine produktive Machtkonstellation auszuweisen, die an bestimmte gesellschaftlich-historische Bedingungen gebunden bleibt und die Reflexion auf das B estehende einer Gesellschaft im Lichte der M oral erlaubt. Auch wenn wir uns beständig in einem Raster reglementierter Normen bewegen müssen, um über haupt als intelligible Subjekte zu gel ten, haben wi r gleichwohl die Möglichkeit, uns zu uns selbst zu verhalten und einen Grad der Freiheit zu erfahren. Die Verbindung von Denken und Moral entspricht dann einem Selbstverhältnis, einer Existenzweise, das sich in der Alterität konstituiert. Mit Blick auf die Hervorbri ngung eines Moralsubjekts nämlich geht Foucault so weit zu behaupten, dass die Einhaltung einer jeglichen Moral sogleich auch eine Überprüfung der eigenen Lebensführung nach sich zieht. Bezogen auf einen präskriptiven Moralcode bedeutet das, dass sich das Subjekt in einem Spannungsverhältnis zum eigenen Moralverhalten befindet und daraus Spielräume für das eigene Handeln gewinnt. Dazu gehört auch, dass die Denktätigkeit niemals verharrt und dass im Denkprozess selbst überschüssige Potenziale freigesetzt werden, die es erlauben, die Richtung des Denkens zu ändern und die Wahrnehmungsfähigkeit auszudifferenzieren. Und so kommt auch Arendt zu dem Ergebni s, dass das Denken sich beständig gegen si ch selbst wendet und damit vermeintlich gültige Wahrheiten in der Schwebe häl t. Das Denken ist, mit den Wort en Arendts, ,selbstzerstörerisch“ (Arendt 1994, S. 134), weil es in gewisser Weise destruktive Wirkung hat auf ,etablierte[.] Kriterien, Werte, Maßeinheiten für Gut und Böse, kurz jene Sitten und Regeln des Betragens, die wir in Moral und Ethik behandeln“ (Arendt 1994, S. 143). In diesem Sinne sei Denken ni cht das Pri vileg einzelner, sondern das Geschäft aller und ,eine unentbehrliche Vorberei tung für die Entscheidung darüber [...], was sein soll, und für die Beurteilung dessen, was ni cht mehr i st" (Arendt 2002, S. 209). Nicht-Denken verführe dagegen zu moralischer Indifferenz. ${ }^{1}$

Noch ein Weiteres drückt sich darin aus. Denn das Denken ist nicht etwa durch ein Kon tinuum von Vergangenheit, Gegenwart und Zukunft gekenn-

1 Ein wesentlicher Bezugspunkt von Arendts politischer Philosophie ist die Shoa. Dabei spielt die Frage nach dem Zusammenhang von Denken und Moral eine zentrale Rolle. 
zeichnet. Ganz im Gegenteil: „Diese Vergangenheit, die ganz weit zurück bis an den Ursprung reicht, zieht nicht nach rückwärts, sondern drückt vorwärts, und im Gegensatz zu dem, was man erwarten würde, ist es die Zukunft, die uns in die Vergangenheit zurücktreibt. Aus der Sicht des Menschen, der immer in dem Zwischenbereich zwischen Vergangenheit und Zukunft lebt, ist die Zeit nicht ein Kontinuum, nicht ein Fluß von ununterbrochen Aufeinanderfolgendem; sie ist in der Mitte, dort, wo ,Er' [d er Mensch, E.B.] steht, aufgebrochen; und ,sein' Standort ist nicht die Gegenwart, wie wir sie gewöhnlich verstehen, sondern vielmehr eine Lücke in der Zeit, die von ,seinem' dauernden Kämpfen, , seinem“ Standpunkt-Beziehen gegen die Vergangenheit und Zukunft aufrechterhalten wird" (Arendt 1994, S. 14). ${ }^{2}$

Diese Lücke in der Zeit ist ein wesentliches Moment des Denkens, sie ist unvermeidliche Voraussetzung dafür, dass sich das Denken ausbreiten und wieder bündeln kann. Dem steht eine schnelle und effiziente Wissensproduktion diametral entgegen. Weil Beschleunigung dazu angetan ist, die erforderliche Lücke zwischen Erfahrungsraum und Erwartungshorizont zusammenschnurren zu lassen, liegt in ihr auch eine grundsätzliche Gefährdung des Denkens. Andererseits: Was das Denken erst in Gang setzt, ist die ihm eigene, unablässige Herausforderung durch sich selbst. Indem es sich beharrlich fremd wird, vollzieht es sich im Spannungsfeld der Alterität, im Unterschied, in der Differenz. Insofern ist die Gefährdung im Denken die produktive Seite des Denkens überhaupt, oder, wie Arendt notiert: „Es gibt keine gefährlichen Gedanken; das Denken selbst ist gefährlich“ (Arendt 1994, S. 144).

Alterität -Weibliche Intellektualität gewinnt sich nicht nur in der Weise, dass sie über ihre eigenen Konstitutionsbedingungen reflektiert, sondern sie bringt sich über ein Denken hervor, das sich selbst zum Gegenstand wird und darin sein kritisches Potenzial erhält. Ein Nachdenken über das Denken als einer für die (epistemologische) Theoriebildung notwendigen Tätigkeit, einer Arbeit, die sich nicht an Zeit und Raum hält und auch kein Gegenstand einseitiger Verbindlichkeiten sein kann, muss sich darüber klar sein, dass die kulturellen Verlockungen mimetischer Angleichung dem Denken selbst feindlich sind. Weil die Differenz zum Ausgangspunkt des Denkens wird, weil sich weibliche Intellektualität gerade in der Differenz zur symbolischen Ordnung entwirft, müsste sie zu einem unumgänglichen Ort werden: Die Differenz im dialektischen Verhältnis zwischen Innen und Außen, zwischen Einschluss und Ausschluss, zwischen Verworfenem und Akzeptiertem wird so zum ent-

2 Eine ähnliche Denkfigur entfaltet übrigens Walter Benjamin in Gestalt seines Engels der Geschichte (Angelus Novus). 
scheidenden Kriterium der Kritik und zum Zeichen einer (weiblichen) Identität, die sich an den eigenen und den fremdbestimmten Verwerfungen erzeugt und sich darum erst als ebenso produktiv wie machtvoll im politischen Spiel der Mächte erweist. In dieser Alterität liegt auch, wie Marianne Schuller notiert, ,der Ausgangspunkt für die Ausbildung einer weiblichen Intellektualität, die nicht mehr gezwungen wäre, sich über das mächtige ,Andere ihres Selbst“ herzustellen“. Alterität könnte vielmehr „in das Selbst als komplexe heterogene Struktur" eingehen" (Schuller 1990, S. 194), und zwar bei beiden Geschlechtern. Der Abschied vom emphatischen Selbst bedeutet dann eine Kritik am Selbst, die die Möglichkeit eröffnet, das Andere des Anderen in seiner besonderen Form anzuerkennen.

Frauen und Macht - Unter den Bedingungen der Alterität in der doppelten Art, dass sie das Denken im Unterschied hervorbringt und zugleich der Unterschied zum signifikanten Zeichen der Kritik aufrückt, kann sich Macht nicht verstetigen. Sie ist in den Prozess einer unaufhörlichen Bewegung eingeschlossen, deren Ursache und deren $\mathrm{R}$ esultat sie gleichermaßen ist. Einmal produktiv geworden vermag sie zwar hegemoniale Diskurse hervorzubringen. Sie kann aber ebenso den Versuch der totalisierenden Vereinnahmung unterlaufen und reduzierende Identitätsvorgaben zurückweisen, politische und moralische Haltungen als eine kritische Denkungsart hervorbringen und den Ort der Unverfügbarkeit markieren, jenen Rest, der sich im Recht auf Andersheit artikuliert.

Wie wir gesehen haben, scheinen sich derzeit Verschiebungen sowohl im politisch-gesellschaftlichen Raum wie auch in den Wissenschaftsauffassungen selbst anzukündigen, von denen Frauen in einer besonderen Wei se affiziert sind. Zwei diskursive Muster, die sich sowohl in sozialen Praktiken wie auch in Form affirmativer Selbsttechnologien niederschlagen, sind dabei ausschlaggebend: eine konservative, weil naturalisierende Vorstellung von Geschlecht und der Versuch, die Gesellschaft mit ihren Organisationen und Institutionen nach dem Vorbild eines wirtschaftlich erfolgreichen Unternehmens zu modeln und diesem Modell den Anschein alternativloser Gültigkeit zu verleihen. Das Zusammentreffen dieser beiden Diskurse, ihre wechselseitige Verschränkung und ihre Koinzidenz in Hinsicht auf i hre vorgebliche Naturwüchsigkeit geben zu der Überlegung Anlass, dass sich Fragen nach dem kul turellen Ort der Frauen, ihrer Identität und ihrer Intellektualität mit einer Kritik der - darin schon immer eingelassenen - politischen Ökonomie verbinden muss.

Die diskursive Überschneidung macht sich augenblicklich gerade dort bemerkbar, wo es um die Verteilung von Wissen und Macht geht, dort also, wo sich weibliche Intellektualität in besonderer Weise auszuprägen vermag: 
an der Universität. So, wie es aussieht, ist kein Ende des „akademischen Frauensterbens" (Hassauer) erkennbar, denn abweichende Geschlechtsidentität, abweichende Biographiemuster, abweichende Vereinbarkeit von Privatbereich und Karriere, abweichende Zeitökonomie, abweichender Habitus und abweichendes symbolisches Kapital sind allemal dazu angetan, das traditionell homosoziale Patronatssystem aufrecht zu erhalten, wie Friederike Hassauer in ihrer Antrittsvorlesung von 1993 diagnostiziert hatte (vgl. Hassauer 1994, S. 35 ). Nicht absehen konnte sie, dass dieses System 13 Ja hre später unter einen ökonomischen Druck gerät, der auch abweichendes Alter, abweichende Lehrmeinungen und theoretische Verortungen schon zum Problem werden lassen. Diejenige, die Geschlechtertheorie betreibt, ist out; diejenige, die es vorzieht, sich auf anderen theoretischen Feldern zu tummeln, ist deshalb aber noch nicht in, zumal, wenn dekretiert wird, sie sei nicht einschlägig genug oder sie sei gar zu alt.

Der Versuch, die eigene Stimme im Modus der Alterität als heterogene Struktur des Selbst zum Ausdruck kommen zu lassen, sie laut, vernehmlich und öffentlich zu erh eben und dabei - unumgänglich - im Kontext von Grenzüberschreitungen das eigene Selbst probeweise zu riskieren, ist bislang nur kontrafaktisch und als theoretisches Gedankenexperiment vorstellbar. Der tägliche „Affentanz“ in Gestalt von „Maskierung-Mimikry-Parodie-Subversion [...] unter den Bedingungen von social grace und Lächelzwang“ (Hassauer 1994, S. 34) ist also nicht vorüber. Eher im Gegenteil. Denn in der intrikaten Verschränkung der genannten Diskurse, die ihren konstruktiven Charakter in der strategischen Verbindung mit wissenschaftlichem Expertentum zu verschleiern trachten und Auswirkungen auf die sozialen, kulturellen und politischen Praktiken haben, drückt sich mehr denn je das aus, was intellektuellen Frauen seit jeher das Leben schwer macht: die Aporie von gleichzeitigem Ein- und Ausschluss unter der dominanten Deutungshoheit des repräsentativen Sprechens in der symbolischen Ordnung. Intellektualität hat nämlich immer noch für die weibliche Identität den Status einer Gabe: „Sie wird den Frauen ge- oder verabreicht wie die Oblate aus der Hand des Priesters“"(Schuller 1990, S. 192).

Was bleibt - Übungen im politischen Denken: Wissen(schaft)s- und Machtkritik, Kritik von Identitätspolitiken und Kritik der politischen Ökonomie, beharrlich vorgetragen und unbedingt in ihrem Anspruch auf Gehör, diesseits und jenseits der Universitätsmauern, ausgestattet mit der Lust, dem Begehren, der Begierde, dem Randständigen zur Geltung zu verhelfen.

Dazu gehört der Mut zur klug kalkulierten Unfügsamkeit. 


\section{Literatur}

Arendt, Hannah (1994): Zwischen Vergangenheit und Zukunft. Übungen im politischen Denken I, München

Arendt, Hannah (2002): Vom Leben des Geistes. Das Denken. Das Wollen, München Bourdieu, Pierre (1998): Der Beruf der Wissenschaft und die soziale Bew egung, in: ders.: Gegenfeuer, Konstanz, S. 60-67

Derrida, Jacques (2001): Die unbedingte Universität, Frankfurt am Main

Foucault, Michel (1987): Gespräch zwisch en Michel Foucaul t und Gilles Deleuze. Die Intellektuellen und die Macht, in: ders.: Von de r Subversion des Wissens, Frankfurt am Main, S. 106-115.

Hassauer, Friederike (1994): Homo. Acade mica. Geschlechterkontrakte, Institution und die Verteilung des Wissens, Wien

Schuller, Marianne (1990): Vergabe des Wissens. Notizen zum Verhältnis von, weiblicher Intellektualität" und Macht, in: dies.: Im Unterschied, Frankfurt am Main, S. $189-198$ 